\title{
Epidural anesthesia for cesarean section in a patient with Marfan syndrome and dural ectasia
} -A case report-

\author{
Gahyun Kim, Justin Sangwook Ko, and Duck Hwan Choi
}

Department of Anesthesiology and Pain Medicine, Samsung Medical Center, Sungkyunkwan University College of Medicine, Seoul, Korea

Pregnancy is considered a period of high risk for cardiovascular complications in patients with Marfan syndrome. Therefore the choice of anesthetic technique for delivery should be focused on minimizing hemodynamic fluctuations, and preferably provide adequate post-operative pain control. For this purpose, neuraxial blocks, such as spinal or epidural anesthesia, may be deemed a safe option. However, dural ectasia is present in $63-92 \%$ of patients with Marfan syndrome, and the increased amount of cerebrospinal fluid volume is thought to be one of main reasons for spinal anesthesia failure. We report herein the peri-operative management of a patient with Marfan syndrome and dural ectasia for cesarean section using epidural anesthesia. (Korean J Anesthesiol 2011; 60: 214-216)

Key Words: Cesarean section, Epidural anesthesia, Marfan syndrome, Pregnancy.

Marfan syndrome is an autosomal dominant inherited connective tissue disease [1]. Marfan syndrome involves many organ systems, but the cardiovascular manifestations, such as aortic dilation and dissection, are responsible for $90 \%$ of deaths attributed to Marfan syndrome [2,3]. Pregnancy is considered to be a period of high risk for cardiovascular complications, such as aortic dissection, and thus the choice of anesthetic technique is particularly important in patients with Marfan syndrome [4]. Combined spinal-epidural anesthesia is preferred over general anesthesia for cesarean section in patients with Marfan syndrome because combined spinal-epidural anesthesia provides excellent hemodynamic stability and adequate post- operative pain control may be obtained via epidural analgesia. However, several cases of spinal anesthesia failure have been reported in Marfan patients, possibly due to dural ectasia [5]. Dural ectasia is a ballooning or outpouching of the dural sac, primarily involving the lower lumbar and sacrum, and is present in greater than two-thirds of affected adults (63$92 \%$ ). The most common clinical symptoms of dural ectasia are low back pain, headache, weakness, loss of sensation above and below the affected limb, and occasional rectal or genital pain [6]. Greater than a normal volume of cerebrospinal fluid (CSF) in the lumbar theca is postulated to restrict the spread of intrathecally-injected local anesthetic and is thought to be one

Received: September 13, 2010. Revised: October 5, 2010. Accepted: October 7, 2010.

Corresponding author: Duck Hwan Choi, M.D., Department of Anesthesiology and Pain Medicine, Samsung Medical Center, Sungkyunkwan University College of Medicine, 50, Ilwon-dong, Gangnam-gu, Seoul 135-710, Korea. Tel: 82-2-3410-2466, Fax: 82-2-3410-0369, E-mail: dhchoi@skku.edu (c) This is an open-access article distributed under the terms of the Creative Commons Attribution Non-Commercial License (http:// creativecommons.org/licenses/by-nc/3.0/), which permits unrestricted non-commercial use, distribution, and reproduction in any medium, provided the original work is properly cited. 
of the main reasons for spinal anesthesia failure [7]. Herein we report the peri-operative management of a patient with Marfan syndrome with dural ectasia for cesarean section using epidural anesthesia.

\section{Case Report}

A 35-year-old woman with Marfan syndrome was admitted at 37 weeks gestational age (GA). She was diagnosed with Marfan syndrome at 16 years of age. A previous pregnancy at 33 years of age culminated in a missed abortion at 6 weeks GA, for which a dilatation and curettage was performed at our hospital under monitored anesthesia with propofol and remifentanil. She underwent a Bentall operation due to aortic regurgitation at 20 years of age. She underwent a vitrectomy at 27 years of age for subluxation of the right lens. She had orthostatic headaches 3 years ago, and dural ectasia at the 5th lumbar and sacrum, which was identified on lumborsacral spine magnetic resonance myelography, was thought to be the cause of the symptoms. At the time of admission, she had a $35-\mathrm{mm}$ abdominal aortic aneurysm for 8 years. The cardiac functions were stable because the previous aortic surgical procedures were well-maintained during the current pregnancy. She was medicated with verapamil, atenolol, and warfarin, which were switched to labetalol and enoxaparin during the pregnancy.

She had the pathognomic body habitus of Marfan syndrome (height, $179 \mathrm{~cm}$; weight, $76.2 \mathrm{~kg}$; and arachnodactyly). A metallic click and systolic murmur were auscultated with a stethoscope. Her initial vital signs showed a blood pressure of 130/70 $\mathrm{mmHg}$, a heart rate of $80 / \mathrm{min}$, and an oxygen saturation of $100 \%$ on room air. A pre-operative echocardiography revealed a normal left ventricle ejection fraction (55-60\%) and a well-functioning prosthetic aortic valve. Mild ectatic changes in the proximal descending thoracic aorta and distal abdominal aorta $(32 \times 36$ and $34 \times 31 \mathrm{~mm}$, respectively) were confirmed with no interval changes compared to the last study checked 1 year ago.

An elective cesarean section was recommended by the obstetricians, cardiologists, and anesthesiologists involved in the patient's care. Combined spinal-epidural anesthesia is the standard anesthetic choice for cesarean section at our hospital. However, the presence of dural ectasia at the 5th lumbar and sacrum, and a neurosurgeon's warning against spinal anesthesia for the possible recurrence of headache associated with CSF leakage, led us to decide on epidural anesthesia. Enoxaparin was discontinued one day before the scheduled cesarean section and unfractionated conventional heparin was administered until 6 hours before the operation.

With the patient in the right lateral position, a 17-gauge Tuohy needle was inserted at the L3-4 interspace under sterile conditions using a midline approach with the loss-of- air resistance technique. Then, a 19-gauge epidural catheter (FlexTip Plus ${ }^{\circledR}$; Arrow, USA) was inserted via the Tuohy needle and fixed at $9 \mathrm{~cm}$. Eight $\mathrm{ml}$ of $2 \%$ lidocaine with epinephrine $(1: 200,000)$ and fentanyl $(50 \mu \mathrm{g})$ were injected via an epidural catheter as a divided first dose, and the total $27 \mathrm{ml}$ of $2 \%$ lidocaine with epinephrine $(1: 200,000)$ and fentanyl $(100 \mu \mathrm{g})$ was injected incrementally over 10 minutes. Twenty minutes after the last epidural injection of lidocaine, the sensory block was sufficiently high (T4) for the cesarean section. The systolic blood pressure was decreased by $25 \%$ and 3 doses of phenylephrine $(100 \mu \mathrm{g})$ and 1 dose of ephedrine $(10 \mathrm{mg})$ were administered to maintain the blood pressure at 100/50 $\mathrm{mmHg}$. She was sedated with propofol after the infant was delivered and transferred to the post-anesthesia care unit. The baby had been diagnosed prenatally with possible Marfan syndrome and was transferred to the neonatal intensive care unit. The patient's post-operative vital signs were stable with a blood pressure of $106 / 68 \mathrm{mmHg}$, a heart rate of $83 / \mathrm{min}$, and an oxygen saturation of $100 \%$. Heparin was re-infused 6 hours after the cesarean section. The post-operative pain was managed with patientcontrolled epidural analgesia for 3 days post-operatively. The epidural catheter was removed 6 hours after stopping the continuous heparin injection on the 3rd post-operative day. She was transferred to the Cardiology Department for postpartum management and discharged without cardiovascular complications.

\section{Discussion}

Pregnancy is considered to be a period of high risk for cardiovascular complications, such as aortic dilatation and dissection, in patients with Marfan syndrome [4]. A number of studies have stratified the risk for a rapid change in aortic size or aortic tear during pregnancy or immediately after delivery according to the degree of aortic root dilatation. $[2,8]$. Patients with an aortic root $<4 \mathrm{~cm}$ in diameter at the time of delivery have a similar outcome for vaginal and cesarean section delivery, but cesarean section is preferred in patients with an aortic root dilatation $>4 \mathrm{~cm}$ because the risk for cardiac decompensation is extremely high [8]. High blood pressure tends to develop aortic aneurysms due to a weakened vascular media in patients with Marfan syndrome. Myocardial ischemia and heart failure can also be caused by an increased myocardial oxygen demand resulting from high blood pressure. Therefore, the most cautious goal is to prevent high blood pressure [9].

In our case, the patient had already undergone aortic replacement and her pre-operative echocardiogram revealed a normal ejection fraction, a well-functioning prosthetic aortic valve, and a descending aortic diameter of $3.5 \mathrm{~cm}$. Based on these clinical findings, both vaginal delivery and cesarean 
section were considered appropriate. However, aortic dissection in gravidas with Marfan syndrome has been reported, even in the absence of pre-existing aortic root dilatation [8], and the fluctuations in hemodynamic parameters secondary to pain and anxiety of labor may have negative effects on the cardiovascular system. Therefore, an elective cesarean section was planned for our patient. Epidural anesthesia was considered first because of the slow onset and gradual progression of epidural block may be more favorable in mitigating hemodynamic fluctuations. In addition, our patient had dural ectasia at the L4-5 level. A larger than normal volume of CSF in the lumbar theca is considered to restrict the spread of intrathecally-injected anesthetics and lead to an increased rate of spinal anesthesia failure [7]. For example, Lacassie et al. [5] reported two cases of inadequate spinal anesthesia in two parturients with Marfan syndrome. They performed continuous spinal anesthesia with an incrementally-increased dose of bupivacaine, but they stopped further administration of bupivacaine after $21 \mathrm{ml}$ for the fear of potential neurologic injury. Further, they suggested that dural ectasia and the associated increase in CSF volume were possible causes of erratic spread of spinal anesthesia.

Although dural ectasia is not an absolute contraindication for epidural anesthesia, the use of epidural anesthesia in patients with Marfan syndrome with moderate-to-severe dural ectasia is not recommended because of the risk of a spinal CSF leak in the case of accidental dural puncture [10]; however, when performed carefully by an experienced anesthesiologist, many benefits of epidural anesthesia, including minimal hemodynamic fluctuations and excellent post-operative pain control, outweigh the potential risk of dural puncture in patients with Marfan syndrome. The weakened connective tissues in patients with Marfan syndrome affect skeletal anomalies and abnormal joint flexibility, and the positional change for regional anesthesia might damage the tissues [9]. In our patient, the surgically-adequate level of anesthesia was achieved $30 \mathrm{~min}$ after the epidural injection of $27 \mathrm{ml}$ of $2 \%$ lidocaine with epinephrine (1:200,000) and fentanyl (100 $\mu \mathrm{g})$. The gradual decrease in blood pressure was well-managed with a bolus injections of phenylephrine and ephedrine. In addition, patient-controlled epidural analgesia provided excellent pain management for 3 days post-operatively.

In summary, we provided epidural anesthesia for cesarean section without any cardiovascular and neurologic complications in a patient with Marfan syndrome, even though she has lumbosacral dural ectasia. Epidural anesthesia may be considered as one of the useful anesthetic options for cesarean section in Marfan syndrome patients with dural ectasia.

\section{References}

1. Elkayam U, Ostrzega E, Shotan A, Mehra A. Cardiovascular problems in pregnant women with the Marfan syndrome. Ann Intern Med 1995; 123: 117-22

2. Paternoster DM, Santarossa C, Vettore N, Dalla Pria S, Grella P. Obstetric complications in Marfan's syndrome pregnancy. Minerva Ginecol 1998; 50: 441-3.

3. Beighton P, de Paepe A, Danks D, Finidori G, Gedde-Dahl T, Goodman R, et al. International nosology of heritable disorders of connective tissue, Berlin, 1986. Am J Med Genet 1988; 29: 581-94.

4. Summers KM, West JA, Peterson MM, Stark D, McGill JJ, West MJ. Challenges in the diagnosis of Marfan syndrome. Med J Aust 2006; 184: 627-31

5. Lacassie HJ, Millar S, Leithe LG, Muir HA, Montaña R, Poblete A, et al. Dural ectasia: a likely cause of inadequate spinal anaesthesia in two parturients with Marfan's syndrome. Br J Anaesth 2005; 94: 5004.

6. Ahn NU, Sponseller PD, Ahn UM, Nallamshetty L, Kuszyk BS, Zinreich SJ. Dural ectasia is associated with back pain in Marfan syndrome. Spine (Phila Pa 1976) 2000; 25: 1562-8.

7. Fettes PD, Jansson JR, Wildsmith JA. Failed spinal anaesthesia: mechanisms, management, and prevention. Br J Anaesth 2009; 102: $739-48$.

8. Rahman J, Rahman FZ, Rahman W, al-Suleiman SA, Rahman MS. Obstetric and gynecologic complications in women with Marfan syndrome. J Reprod Med 2003; 48: 723-8.

9. Kim DW, Lim YG. A case report of marfan syndrome under general anesthesia. Korean J Anesthesiol 1993; 26: 1055-8.

10. Buser RT, Mordecai MM, Brull SJ. Combined spinal-epidural analgesia for labor in a patient with Marfan's syndrome. Int J Obstet Anesth 2007; 16: 274-6. 